\title{
Tandem queueing networks with neighbor blocking and back-offs
}

\author{
Ton Hellings • Sem C. Borst • \\ Johan S.H. van Leeuwaarden
}

Received: 9 May 2011 / Revised: 9 May 2011 / Published online: 1 July 2011

(C) The Author(s) 2011. This article is published with open access at Springerlink.com

\begin{abstract}
We introduce a novel class of tandem queueing networks which arise in modeling the congestion behavior of wireless multi-hop networks with distributed medium access control. These models provide valuable insight in how the network performance in terms of throughput depends on the back-off mechanism that governs the competition among neighboring nodes for access to the medium. The models fall at the interface between classical queueing networks and interacting particle systems, and give rise to high-dimensional stochastic processes that challenge existing methodologies. We present various open problems and conjectures, which are supported by partial results for special cases and limit regimes as well as simulation experiments.
\end{abstract}

Keywords Wireless networks · Tandem queues · Neighbor blocking · Interacting particle systems $\cdot$ Stability $\cdot$ Fluid limit $\cdot$ High-dimensional stochastic processes

Mathematics Subject Classification (2000) 60K25 - 60J27 · 82C22

\footnotetext{
T. Hellings · S.C. Borst $(\bowtie) \cdot$ J.S.H. van Leeuwaarden Department of Mathematics and Computer Science, Eindhoven University of Technology, P.O. Box 513, 5600 MB Eindhoven, The Netherlands e-mail: s.c.borst@tue.nl

T. Hellings

e-mail: tonhellings@gmail.com

J.S.H. van Leeuwaarden

e-mail: j.s.h.v.leeuwaarden@tue.nl

S.C. Borst

Alcatel-Lucent Bell Labs, Murray Hill, USA

J.S.H. van Leeuwaarden

EURANDOM, Eindhoven, The Netherlands
} 


\section{Introduction}

Motivated by wireless networks with distributed medium access control, we introduce a novel class of models at the interface between classical queueing networks and interacting particle systems. Specifically, we focus on tandem networks in which data packets are transferred from a source to a receiver via several relay nodes. The feature that distinguishes these networks from their traditional counterparts is that an active node that is transmitting a packet, 'blocks' its neighbors and prevents them from being active. Upon completion of the transmission, a node is forced into back-off, during which it is not allowed to become active, thus granting its neighbors a chance to transmit.

The above-described tandem networks with neighbor blocking and back-offs arise as models for wireless networks with distributed medium access control, particularly the so-called Carrier-Sense Multiple-Access Collision Avoidance (CSMA/CA) protocol. In CSMA/CA, each node attempts to access the medium, but nodes that sense the medium busy postpone their attempt until the medium is sensed idle.

Although widely deployed in practice, the CSMA/CA protocol is prone to severe performance problems in multi-hop networks $[1,12,14]$. In particular, nodes in the 'middle' of a network tend to experience more interference than nodes on the 'edge' of the network and, therefore, are at a disadvantage in competing for medium access. This issue, known as the node-in-the-middle problem, can cause extreme unfairness and starvation effects, manifesting themselves in poor throughput and severe congestion. In order to remedy this issue and ensure a smoother flow of packets in multi-hop networks, various forms of flow control have been proposed; see [15] for an overview.

The models in the present paper pertain to a scheme referred to as extra back-off flow control. In this scheme, a node remains silent for a certain extra back-off time (imposed on top of the usual back-off time that is part of the CSMA/CA protocol) after it has transmitted a packet, so as to give both the downstream and upstream neighbors the opportunity to transmit.

We assume the source node to be saturated, but explicitly account for the queueing dynamics at the other nodes, which is especially crucial since these act as relays in transferring the flow of packets. Unfortunately, however, the queueing dynamics severely complicate the mathematical analysis, and give rise to high-dimensional stochastic processes with infinite state spaces, which generally do not admit closedform expressions for the stationary distribution or yield to standard numerical techniques or brute-force simulation, even under Markovian assumptions. In view of the complexity, we focus on a regime where the ordinary back-off periods are asymptotically small. We refer to [4, 11, 13, 21, 25, 27] which consider CSMA/CA networks with standard back-offs only where all nodes are assumed to be saturated. Stability issues in non-saturated networks with standard back-offs and single-hop traffic are pursued in [26].

The feature of neighbor blocking is reminiscent of asymmetric simple exclusion processes (ASEP) from statistical mechanics; see, for instance, [17]. On a onedimensional lattice, particles hop to their right, at exponential times, provided that the target site is empty. The term exclusion refers to the fact that transitions are only allowed to empty sites and all other transitions are suppressed. ASEP is a popular model 
for a driven lattice gas of hard-core particles with nearest-neighbor interaction. There are crucial differences between our model and ASEP, though. The nearest-neighbor interaction in ASEP is biased and limited in range: a particle is only blocked by a particle to its immediate right. Our model has a two-sided nearest-neighbor interaction, so that particles can be blocked by particles at either side, and the interaction can extend across several neighboring nodes.

A further key difference is that in ASEP each site can be occupied by at most one particle, whereas in our model the nodes are endowed with buffers to store any number of packets. Due to the possible accumulation of packets, the queueing dynamics strongly impact the flow of traffic through the network. If all nodes were assumed to be saturated, and thus have always packets to transmit, then the queueing dynamics would not play a role. In fact, under these assumptions connections between queueing networks and interacting particle systems, including ASEP, were exploited in [24] to investigate certain time-dependent characteristics of large Jackson tandem networks (infinite series of exponential queues). Tandem queues with interaction have also been studied in the context of manufacturing flow line systems [5, 10], in which blocking occurs at times when the (finite-size) buffers of neighboring downstream nodes become full. Blocking through buffers in linear wireless networks has been investigated in $[16,20]$.

The remainder of the paper is organized as follows. In Sect. 2 we present a detailed model description. In Sect. 3 we discuss the main conjecture, and provide support in the form of partial results for special cases and limiting regimes as well as simulation experiments. A brief outlook is given in Sect. 4.

\section{Model description}

We now present a more specific model description. Consider a tandem network of $N$ nodes, in which packets are transferred from the source node 1 to some receiver, via relay nodes $2, \ldots, N$. Assume that the source node 1 is saturated, i.e., always has packets pending for transmission. The relay nodes $2, \ldots, N$ are each endowed with a buffer of infinite size. All transmission times are independent and exponentially distributed with unit mean. When transmitting, a node always 'blocks' its $k$ neighboring nodes on either side, so that two nodes $i$ and $j$ can only be active simultaneously if $|i-j|>k$. The value of $k$ is referred to as the interference range. Upon completion of a transmission, a node is forced into back-off, during which time it is not allowed to be active. All back-off periods are independent and exponentially distributed with mean $\eta>0$. When a node completes a transmission and starts a back-off period, several neighbors may become unblocked simultaneously. Any race conditions that arise in such situations are resolved uniformly at random.

Note that the present model differs from that in [12, 13, 25, 27], in that nodes only enter back-off once after each transmission, and do not re-enter the back-off mode when they find themselves blocked at the end of a back-off period. Alternatively, the back-offs in the present model may be interpreted as 'extra' back-offs, with the duration of the 'regular' back-offs vanishing to zero.

The above-described mechanism will be referred to as the 'basic' back-off scheme. We will also consider a slightly modified version, called the 'truncated' back-off 
scheme, where a node immediately comes out of back-off when it receives a packet from its upstream neighbor.

For either back-off scheme, the state of the tandem network evolves as a Markov process $\left(X_{2}(t), \ldots, X_{N}(t) ; Y_{1}(t), \ldots, Y_{N}(t)\right)$, with $X_{i}(t)$ denoting the number of packets at node $i$ at time $t$ (including the packet being transmitted, if any) and $Y_{i}(t) \in\{0,1, b\}$ indicating whether node $i$ is inactive, transmitting, or in back-off, respectively. The transitions slightly depend on the version of the back-off scheme that is used, with nodes in back-off mode possibly moving instantly to an active state upon receipt of a packet in the truncated scheme. Note that the state space of the Markov process is infinite in $N-1$ dimensions, and hence the stationary distribution is difficult to determine, except in the (uninteresting) special case of $N=2$. In fact, even deriving stability conditions and throughput values turns out to be a formidable task. For the case of $N=3$ and $k=1$, we obtained in [15] exact and approximative results for the stationary distribution and the throughput using the theory of quasi birth-death processes. However, this approach does not extend to larger networks. Also, the results in [26] demonstrate that even with standard back-offs and singlehop traffic, an explicit characterization of the stability conditions is hard in general, expect in the special case of a complete interference graph.

\section{Stability and throughput}

In order to state the main conjecture, let $\theta_{i}(\eta)$ be the long-term throughput of node $i$ as function of the back-off parameter $\eta$, i.e., the long-term average number of packets transmitted by node $i$ per time unit, or equivalently, the long-term fraction of time that node $i$ is transmitting (assuming it exists). Note that by definition,

$$
\theta_{1}(\eta) \geq \theta_{2}(\eta) \geq \cdots \geq \theta_{N}(\eta)
$$

since the throughput of a node can never exceed that of its upstream neighbor. Also, let $X_{i}^{(m)}(t)$ be the number of packets at time $t$ at node $i$, in a modified version of the network where the first $m$ nodes are saturated, while the remaining $N-m$ nodes receive packets from their upstream neighbors. Let $\theta_{i}^{(m)}(\eta)$ be the corresponding throughput of node $i$. Note that $X_{i}^{(1)}(t)$ and $\theta_{i}^{(1)}(\eta)$ correspond to the original system with only the source node 1 saturated. Define

$$
l:=\min \left\{m: \theta_{m}^{(m)}(\eta)=\theta_{m+1}^{(m)}(\eta)\right\} .
$$

It is easily verified that $l$ is well defined, since

$$
\theta_{N-1}^{(N-1)}(\eta)=\theta_{N}^{(N-1)}(\eta)
$$

Indeed, suppose the latter is not the case, i.e.,

$$
\theta_{N-1}^{(N-1)}(\eta)>\theta_{N}^{(N-1)}(\eta)
$$


then node $N$ must be saturated in addition to nodes $1, \ldots, N-1$. This results in a completely symmetric system, and leads to

$$
\theta_{1}^{(N-1)}=\cdots=\theta_{N}^{(N-1)},
$$

which is a contradiction with the supposition that

$$
\theta_{N-1}^{(N-1)}(\eta)>\theta_{N}^{(N-1)}(\eta)
$$

Conjecture 1 Consider the tandem network with the basic back-off scheme, interference range $k \geq 1$, and $N \geq 2 k+1$ nodes. Then for any back-off parameter $\eta>0$,

(i) $\theta_{1}(\eta)>\theta_{2}(\eta)>\cdots>\theta_{l}(\eta), \theta_{i}(\eta)=\theta_{i}^{(l)}(\eta), i=1, \ldots, l$, and the process $\left(X_{2}(t), \ldots, X_{l}(t)\right)$ is transient, with

$$
\frac{\left(X_{2}(t), \ldots, X_{l}(t)\right)}{t} \rightarrow\left(\Delta_{2}, \ldots, \Delta_{l}\right) \text { as } t \rightarrow \infty,
$$

where $\Delta_{i}:=\theta_{i-1}^{(l)}(\eta)-\theta_{i}^{(l)}(\eta)>0$.

(ii) The process $\left(X_{l+1}(t), \ldots, X_{N}(t)\right)$ is positive-recurrent, which entails $\theta_{l}(\eta)=$ $\theta_{l+1}(\eta)=\cdots=\theta_{N}(\eta)$

(iii) The bottleneck node $l$ is given by $l=k+1$.

Conjecture 1 thus states that node $k+1$ is the bottleneck in the tandem network, in that the throughput values strictly decrease moving downstream up to node $k+1$, implying that nodes $2, \ldots, k+1$ are all 'unstable', while nodes $k+2, \ldots, N$ are 'stable', all having the same throughput as node $k+1$. The intuition is that node $i+1$ is worse off than node $i$ for any $i=1, \ldots, k$, since it has one more upstream neighbor to compete with, while by the same token node $i+1$ cannot be strictly worse off than node $i$ for $i=k+1, \ldots, N-1$.

A quite powerful method for establishing (in)stability properties as in Conjecture 1 is provided by the so-called fluid-limit approach [6-9, 22, 23]. The fluid-limit approach has been successfully applied to derive stability conditions for a wide range of multi-class queueing networks.

In the present situation, the fluid-limit approach would involve deriving a set of linear differential equations $x_{i}^{\prime}(t)=\theta_{i-1}(\eta ; I(x(t)))-\theta_{i}(\eta ; I(x(t)))$, and establishing that $x^{(u)}(t) / t$ tends to the vector $\left(\Delta_{2}, \ldots, \Delta_{k+1}\right)$ as $t \rightarrow \infty$, while $x^{(d)}(t)$ reaches 0 in finite time for any initial state $x(0) \in \mathbb{R}^{N-1}$ with $\|x(0)\|=1$. Here,

$$
\begin{aligned}
x(t) & \equiv\left(x^{(u)}(t), x^{(d)}(t)\right), \\
x^{(u)}(t) & \equiv\left(x_{2}(t), \ldots, x_{k+1}(t)\right), \\
x^{(d)}(t) & \equiv\left(x_{k+2}(t), \ldots, x_{N}(t)\right), \\
I(x) & =\left\{i: x_{i}=0\right\},
\end{aligned}
$$

and $\theta_{j}(\eta ; I), I \subseteq\{2, \ldots, N\}$, represents the throughput of node $j$ in a scenario where the nodes $i \notin I$ are saturated and always compete for access to the medium, while the 
Fig. $1 \theta_{1}(\eta)$ and $\theta_{2}(\eta)=\theta_{3}(\eta)$ for $k=1, N=3$ and the basic back-off scheme

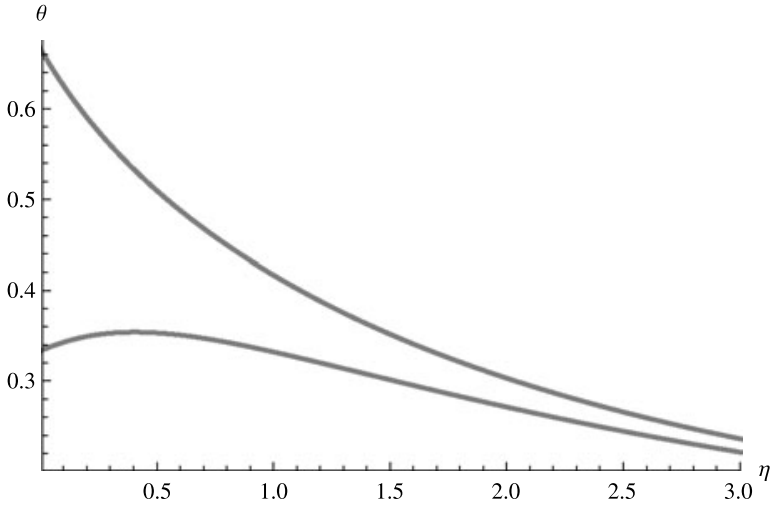

nodes $i \in I$ receive the packets that have been transmitted by their upstream neighbors and only contend for the medium when they have packets pending for transmission, with $\theta_{j}(\eta ;\{m+1, \ldots, N\}) \equiv \theta_{j}^{(m)}(\eta)$ as defined earlier. Note that the process $x(t)$ follows simple piecewise linear trajectories. The difficulty, though, stems from the fact that the coefficients $\theta_{j}(\eta ; I)$ that govern the evolution of the process $x(t)$ involve the stationary distribution of a Markov process with an infinite state space in $|I|$ dimensions. In effect, deriving the stability properties and throughput values is therefore potentially as hard as determining the entire stationary distribution of the stochastic process involved.

The latter is only feasible in special cases. For example, in case $k=1$ and $N=3$, it may be shown that $\theta_{1}(\eta ; I)>\theta_{2}(\eta ; I)$ for any $I \subseteq\{2,3\}$. Thus, the process $\left(x_{2}(t), x_{3}(t)\right)$ cannot spend any amount of time in a region with $x_{2}(t)=0$. Also, $\theta_{2}(\eta ; \varnothing)<\theta_{3}(\eta ; \varnothing)$, so $x_{3}(t)$ must hit 0 eventually. From that point onward, $x_{3}(t)$ will remain 0 while $x_{2}(t)$ will grow at rate $\theta_{1}(\eta ;\{3\})-\theta_{2}(\eta ;\{3\})>0$. This means that node 2 is unstable while node 3 is stable. Note that inequalities for the coefficients $\theta_{j}(\eta ; I)$ suffice for establishing the (in)stability properties. In order to obtain the exact throughput values $\theta_{1}(\eta)=\theta_{1}(\eta ;\{3\})$ and $\theta_{2}(\eta)=\theta_{3}(\eta)=\theta_{2}(\eta ;\{3\})=\theta_{3}(\eta ;\{3\})$, however, we need to find the stationary distribution of a modified system where nodes 1 and 2 are saturated while node 3 receives packets that have been transmitted by node 2 . Now observe that the number of packets $X_{3}(t)$ at node 3 then evolves as a quasi-birth-death process. The stationary distribution of $X_{3}(t)$ may then be numerically obtained using the matrix-analytic method, along with the exact values of $\theta_{1}(\eta ;\{3\})$ and $\theta_{2}(\eta ;\{3\})=\theta_{3}(\eta ;\{3\})$.

Figure 1 shows the throughputs of nodes 1 and 2 calculated from the numerical solution to a quasi-birth-death process in [15]. For small back-off parameters, nodes 2 and 3 benefit, while larger back-offs eventually lead to lower throughputs for all three nodes. Although the throughput values converge to each other rapidly-in fact $\theta_{1}(\eta)-\theta_{2}(\eta)=O\left(\eta^{-3}\right)$, see [15]-they will never be exactly equal, and hence the tandem network will never be stable.

As Conjecture 1 reflects, determining the throughput values is a challenging problem in general. We therefore now proceed to state a few partial results for limit regimes with very small and very large back-off periods, respectively. 
Recall that Conjecture 1 suggests that the basic back-off scheme fails to stabilize the network for any back-off parameter $\eta$. The next proposition confirms this for a network with nearest-neighbor blocking $(k=1)$ and $\eta$ sufficiently small, and states that the same holds for the truncated back-off scheme.

Proposition 2 Consider the tandem network with either the basic or the truncated back-off scheme, interference range $k=1$, and $N \geq 3$ nodes. Denote $\theta_{i}^{*}=$ $\lim _{\eta \downarrow 0} \theta_{i}(\eta), i=1, \ldots, N$. Then

$$
\theta_{1}^{*}=\frac{2}{3}, \quad \theta_{2}^{*}=\cdots=\theta_{N}^{*}=\frac{1}{3},
$$

regardless of whether the basic back-off scheme or the truncated back-off scheme is used.

Proof In the limit as $\eta \downarrow 0$, the system behaves as a system without any extra backoffs, except that a node that has just completed a transmission refrains from competing for the medium in case there are any other contenders.

We first prove that $\theta_{1}^{*}=2 / 3, \theta_{2}^{*}=\theta_{3}^{*}=1 / 3$. We distinguish two cases: (i) $\theta_{2}^{*}>\theta_{3}^{*}$; and (ii) $\theta_{2}^{*}=\theta_{3}^{*}$. In case (i) node 3 is unstable, and hence if $N=3$, node 2 will always lose out to nodes 1 and 3 in the competition for the medium, i.e., $\theta_{2}^{*}=0$, yielding a contradiction. If $N>3$, node 2 can only grab the channel when node 1 finishes a transmission while node 4 is blocking node 3 , so that $\theta_{2}^{*} \leq \theta_{4}^{*}$, which yields a contradiction with the assumption that $\theta_{2}^{*}>\theta_{3}^{*}$ and the fact that $\theta_{4}^{*} \leq \theta_{3}^{*}$ by definition. In case (ii), note that each transmission of node 2 will be followed backto-back by a transmission of node 1 . Also, transmissions of node 1 will end/begin as a Poisson process of rate 1 during periods that node 3 is transmitting. Hence, $\theta_{1}^{*} \geq \theta_{2}^{*}+\theta_{3}^{*}=2 \theta_{2}^{*}$, implying that node 2 is unstable, and that all transmissions of node 1 are of the two types described above, so $\theta_{1}^{*}=2 \theta_{2}^{*}$. The fact that node 1 is saturated implies $\theta_{1}^{*}+\theta_{2}^{*}=1$, and it follows that $\theta_{1}^{*}=2 / 3, \theta_{2}^{*}=\theta_{3}^{*}=1 / 3$.

We now proceed to show that $\theta_{4}^{*}=\cdots=\theta_{N}^{*}=1 / 3$. Suppose not, then there exists a node $i$ with $\theta_{i}^{*}<\theta_{i-1}^{*}=1 / 3$, for some $i=4, \ldots, N$. Since node $i$ is unstable, $\theta_{i}^{*}+\sigma_{i}=1$, with $\sigma_{i}$ denoting the fraction of time that either node $i-1$ or node $i+1$ is active. Because $\sigma_{i} \leq \theta_{i-1}^{*}+\theta_{i+1}^{*}$, we have $\theta_{i-1}^{*}+\theta_{i}^{*}+\theta_{i+1}^{*} \geq 1$, while $1 / 3=$ $\theta_{i-1}^{*} \geq \theta_{i}^{*} \geq \theta_{i+1}^{*}$, which means $\theta_{i-1}^{*}=\theta_{i}^{*}=\theta_{i+1}^{*}=1 / 3$, yielding a contradiction.

The next proposition states that the truncated back-off scheme achieves stability for sufficiently large back-off parameter $\eta$.

Proposition 3 Consider the tandem network with the truncated back-off scheme, interference range $k=1$, and $N \geq 3$ nodes. There exists a critical value $\eta^{*}$ such that for all $\eta \geq \eta^{*}$,

$$
\theta_{1}(\eta)=\theta_{2}(\eta)=\cdots=\theta_{N}(\eta)
$$

Proof Suppose there exists a node $i$ with $\theta_{i}<\theta_{i-1}=\theta_{1} \leq 1 /(1+\eta)$. Since node $i$ is unstable, $\theta_{i}+\tau_{i}+\sigma_{i}=1$, with $\tau_{i}$ denoting the fraction of time that node $i$ spends in 
back-off and $\sigma_{i}$ the fraction of additional time that either node $i-1$ or node $i+1$ is active. Because $\sigma_{i} \leq \theta_{i-1}+\theta_{i+1}$, and $\tau_{i}=\theta_{i} \mathbb{E}\left[V_{i}\right]$, with $V_{i}$ representing the effective back-off time of node $i$, we have $\theta_{i-1}+\theta_{i}\left(1+\mathbb{E}\left[V_{i}\right]\right)+\theta_{i+1} \geq 1$, while $1 /(1+\eta) \geq$ $\theta_{i-1}>\theta_{i} \geq \theta_{i+1}$, yielding $3+\mathbb{E}\left[V_{i}\right] \geq 1+\eta$. Now observe that $V_{i}=\min \left\{B_{i}, U_{i}\right\}$, with $B_{i}$ denoting the nominal back-off duration of node $i U_{i}$ the amount of time until node $i$ receives a packet after entering a back-off period. If node $j<i$ is the right-most upstream node of node $i$ that is active when node $i$ enters the back-off period, then $U_{i}$ is stochastically dominated by $\sum_{h=j}^{i-1} Z_{h}$, with $Z_{h}$ i.i.d. copies of an exponentially distributed random variable with unit mean. Indeed, if nodes $j+$ $1, \ldots, i-1$ are inactive at that moment, they either have no packets or are in backoff. In the worst case each of nodes $j+1, \ldots, i-1$ have to wait for their left-hand neighbor to finish its transmission before starting their first transmission. If none of the upstream nodes of node $i$ is active when node $i$ enters the back-off period, then node 1 must be in back-off and $U_{i}$ is stochastically dominated by $Y_{i}=B_{1}+\sum_{h=1}^{i-1} Z_{h}$. It follows that $\mathbb{E}\left[V_{i}\right] \leq \mathbb{E}\left[\min \left\{B_{i}, Y_{i}\right\}\right] \leq \eta / 2+i-1$. Substitution yields $\eta / 2+i+$ $2 \geq 1+\eta$, i.e., $\eta \leq 2(i+1)$.

This way an upper bound to $\eta^{*}$ is found to be $2(N+1)$.

It may be shown that the statement of Proposition 3 extends to back-offs of the form $\eta B$, with $\mathbb{E}[B]=1$, for any strictly random (non-deterministic) variable $B$. For purely deterministic back-offs, the statement does not hold.

The next proposition, which is taken from [15], provides exact expressions for the critical value $\eta^{*}$ and the throughput $\theta(\eta), \eta \geq \eta^{*}$, for a 3 -node network with nearestneighbor blocking $(k=1)$.

Proposition 4 Consider the tandem network with a truncated back-off scheme, interference range $k=1$, and $N=3$ nodes. Define $\eta^{*}:=\sqrt{5}-1$. If $\eta \leq \eta^{*}$, then the throughput values are given by

$$
\begin{array}{r}
\theta_{1}(\eta)=\frac{8+4 \eta+\eta^{2}}{12+14 \eta+5 \eta^{2}+\eta^{3}}, \\
\theta_{2}(\eta)=\theta_{3}(\eta)=\frac{4+6 \eta+2 \eta^{2}}{12+14 \eta+5 \eta^{2}+\eta^{3}} .
\end{array}
$$

If $\eta \geq \eta^{*}$, then $\theta_{1}(\eta)=\theta_{2}(\eta)=\theta_{3}(\eta)$ and

$$
\theta_{1}(\eta)=\frac{1}{1+\eta+\frac{1}{1+\eta}}
$$

The proof of Proposition 4 relies on the fact that for $\eta<\sqrt{5}-1$, we have $\theta_{1}(\eta)>$ $\theta_{2}(\eta)=\theta_{3}(\eta)$, and hence nodes 1 and 2 are saturated, while node 3 can have at most one packet. Hence, the network can be modeled as a Markov process with a finite state space which has a closed-form solution for the stationary distribution. This solution yields (1) and (2). Observe that $\theta_{1}(\eta) \rightarrow 2 / 3$ and $\theta_{2}(\eta) \rightarrow 1 / 3$ as $\eta \downarrow 0$, confirming Proposition 2. 
Fig. $2 \theta_{1}(\eta)$ and $\theta_{2}(\eta)=\theta_{3}(\eta)$ for $k=1, N=3$ and the truncated back-off scheme

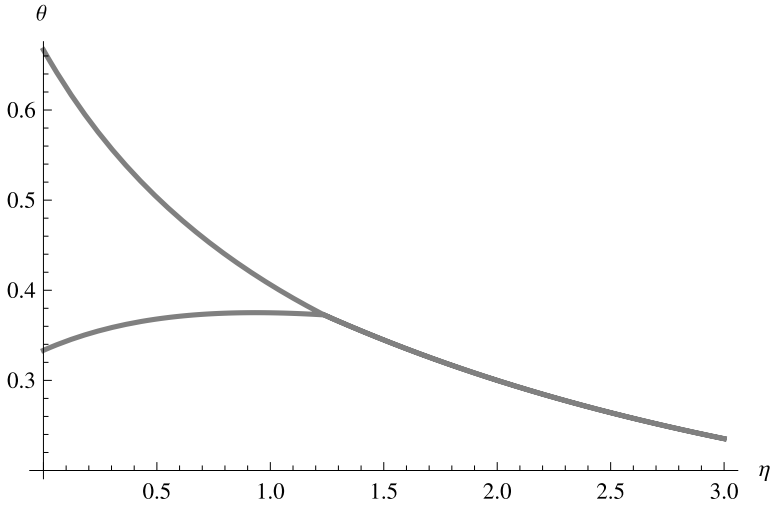

Table 1 Critical values for the truncated back-off scheme and interference range $k=1$

\begin{tabular}{lllllllll}
\hline$N$ & 3 & 4 & 5 & 6 & 7 & 8 & 9 & 10 \\
\hline$\eta^{*}$ & 1.24 & 1.26 & 1.28 & 1.28 & 1.28 & 1.28 & 1.28 & 1.28 \\
\hline
\end{tabular}

For $\eta>\sqrt{5}-1$, only the number of packets at node 2 gives rise to an infinite state space and, therefore, the network can again be modeled as a quasi-birth-death process. The resulting throughput values are plotted in Fig. 2.

For the 4-node network, numerical results can be derived and for networks with 5 nodes or more, results are presented based on extensive simulation experiments. The results suggest that the critical value $\eta^{*}$ only marginally depends on $N$. For $5 \leq$ $N \leq 10$, simulation experiments showed the existence of critical values $\eta^{*}$ beyond which the system remains stable for all values of $N$. The critical values are shown in Table 1 . These values seem to settle around $\eta^{*} \approx 1.28$, suggesting that nodes $5,6, \ldots$ have little impact on the dynamics of the network.

\section{Outlook}

We have introduced a novel class of tandem networks at the interface between classical queueing networks and interacting particle systems. The models involved give rise to rather intricate stochastic processes, and pose severe challenges to existing methodological frameworks.

We have formulated Conjecture 1 relating to stability properties and throughputs values of the various nodes. A possible fluid-limit approach was sketched, where the set of differential equations contains coefficients that involve the stationary distribution of Markov processes with high-dimensional infinite state spaces. Fortunately, for establishing stability properties, it is only required to prove suitable inequalities for these coefficients, for which elementary arguments suffice in some cases. However, calculating the exact throughput values appears to be essentially as hard as determining the entire stationary distribution of the original stochastic process.

The direct use of Lyapounov techniques [18, 19] for the original process provides an alternative approach for establishing stability properties and possibly deriving throughput bounds. As a further option, it seems worth exploring whether similar 
proof concepts may be exploited as developed in the framework of monotone separable networks $[2,3]$.

The conjectures and numerical results suggest that the throughput values in a network with $N=2 k+1$ nodes yield remarkably accurate estimates for those in larger networks. It would be interesting to develop further theoretical support for that observation. By the same token, networks with an extremely large number of nodes will typically provide accurate throughput estimates for networks with a moderate number of nodes. This creates promising scope for spatial asymptotics, for instance, considering networks where the number of nodes grows infinitely large, and using Markov random-field methods.

Open Access This article is distributed under the terms of the Creative Commons Attribution Noncommercial License which permits any noncommercial use, distribution, and reproduction in any medium, provided the original author(s) and source are credited.

\section{References}

1. Aziz, A., Starobinski, D., Thiran, P.: Elucidating the instability of random access wireless mesh networks. In: Proc. of SECON (2009)

2. Baccelli, F., Foss, S.: On the saturation rule for the stability of queues. J. Appl. Probab. 32(2), 494-507 (1995)

3. Baccelli, F., Foss, S.: Moments and tails in monotone-separable stochastic networks. Ann. Appl. Probab. 14(2), 612-650 (2004)

4. Boorstyn, R., Kershenbaum, A.: Throughput analysis of multihop packet radio. In: Proc. of ICC, pp. 1361-1366 (1980)

5. Buzacott, A., Shanthikumar, J.: Stochastic Models of Manufacturing Systems. Prentice-Hall, New York (1993)

6. Chen, H., Yao, D.D.: Fundamentals of Queueing Networks. Applications of Mathematics (New York), vol. 46. Springer, New York (2000). Performance, Asymptotics, and Optimization, Stochastic Modelling and Applied Probability

7. Dai, J.G.: On positive Harris recurrence of multiclass queueing networks: a unified approach via fluid limit models. Ann. Appl. Probab. 5(1), 49-77 (1995)

8. Dai, J.G.: A fluid limit model criterion for instability of multiclass queueing networks. Ann. Appl. Probab. 6(3), 751-757 (1996)

9. Dai, J.G., Meyn, S.P.: Stability and convergence of moments for multiclass queueing networks via fluid limit models. IEEE Trans. Autom. Control 40(11), 1889-1904 (1995)

10. Dallery, Y., Gershwin, S.: Manufacturing flow line systems: a review of models and analytical results. Queueing Syst. 12(1-2), 3-94 (1992)

11. Denteneer, D., Borst, S., Van de Ven, P., Hiertz, G.: IEEE 802.11s and the philosophers' problem. Stat. Neerl. 62(3), 283-298 (2008)

12. Dousse, O.: Revising buffering in CSMA/CA wireless multihop networks. In: Proc. of SECON (2007)

13. Durvy, M., Dousse, O., Thiran, P.: Modeling the 802.11 protocol under different capture and sensing capabilities. In: Proc. of INFOCOM, pp. 2356-2360 (2007)

14. Gambiroza, V., Sadeghi, B., Knightly, E.: End-to-end performance and fairness in multihop wireless backhaul networks. In: Proc. of ACM MobiCom (2004)

15. Hellings, T., Van Leeuwaarden, J., Borst, S., Denteneer, D.: Extra back-off flow control in wireless mesh networks. In: Proc. of WiOpt (2010)

16. Jelenković, P.R., Momcilović, P., Squillante, M.S.: Scalability of wireless networks. IEEE/ACM Trans. Netw. 15(2), 295-308 (2007)

17. Liggett, T.M.: Stochastic Interacting Systems: Contact, Voter and Exclusion Processes. Springer, Berlin (1999)

18. Meyn, S.: Control Techniques for Complex Networks. Cambridge University Press, Cambridge (2008) 
19. Meyn, S., Tweedie, R.L.: Markov Chains and Stochastic Stability, 2nd edn. Cambridge University Press, Cambridge (2009). With a prologue by Peter W. Glynn

20. Momcilović, P., Squillante, M.S.: On throughput in linear wireless networks. In: Proc. of ACM Symposium on Mobile Ad Hoc Networking and Computing. ACM, New York (2008)

21. Rajagopalan, S., Shin, J.: Shah, D. Network adiabatic theorem: An efficient randomized protocol for contention resolution. In: Proc. of ACM SIGMETRICS/Performance, pp. 133-144 (2009)

22. Robert, P.: Stochastic Networks and Queues, vol. 52. Springer, Berlin (2003)

23. Rybko, A.N., Stolyar, A.L.: On the ergodicity of random processes that describe the functioning of open queueing networks. Probl. Pereda. Inf. 28(3), 3-26 (1992)

24. Srinivasan, R.: Queues in series via interacting particle systems. Math. Oper. Res. 18(1), 39-50 (1993)

25. Van de Ven, P.M., Van Leeuwaarden, J.S.H., Denteneer, D., Janssen, A.J.E.M.: Spatial fairness in wireless multi-access networks. In: Proc. of ValueTools (2009)

26. Van de Ven, P.M., Borst, S., Van Leeuwaarden, J., Proutiere, A.: Insensitivity and stability of randomaccess networks. Perform. Eval. 67(11), 1230-1242 (2010)

27. Wang, X., Kar, K.: Throughput modelling and fairness issues in CSMA/CA based ad-hoc networks. In: Proc. of INFOCOM, pp. 23-34 (2005) 